\title{
The glucose level linkages with breast cancer markers
}

Samit Karmakar ${ }^{1}$, Ishita Saha ${ }^{1}$, Ranjan Kumar Sahoo ${ }^{2}$, Sunit Kumar Medda ${ }^{3}$, Rajyalakshmi Kottapalli ${ }^{4}$ and Rabindra Nath Das ${ }^{5^{*}}$

${ }^{1}$ Department of Physiology, Medical College and Hospital, Kolkata, W.B., India

${ }^{2}$ Shool of Statistics, G. M. University, Sambalpur, Odisha, India

${ }^{3}$ Kalyani J.N.M. Hospital, Kalyani, Nadia, West Bengal, India

${ }^{4}$ Dept. of Mathematics, Koneru Lakshmaiah Education Foundation, Vaddeswaram, Guntur, A.P., India

${ }^{5}$ Department of Statistics, The University of Burdwan,Burdwan, West Bengal, India

* Corresponding Author: Rabindra Nath Das, Department of Statistics, The University of Burdwan,Burdwan, West Bengal, India.

Received date: May 05, 2021; Accepted date: May 10, 2021; Published date: May 13, 2021

Citation: S Karmakar, I Saha, Ranjan K Sahoo, Sunit K Medda, R Kottapalli and R Das. (2021) The glucose level linkages with breast cancer markers. J.Cancer Research and Cellular Therapeutics. 5(2); Doi: 10.31579/2640-1053/083

Copyright: (C) 2021, RabindraNath Das, This is an open access article distributed under the Creative Commons Attribution License, which permits unrestricted use, distribution, and reproduction in any medium, provided the original work is properly cited.

\begin{abstract}
:
The glucose level linkages with breast cancer $(\mathrm{BC})$ biomarkers are focused in the article adopting probabilistic modeling with a real data set surveyed from $64 \mathrm{BC}$ women and 52 normal women along with 10 interested study factors. It is derived that mean glucose levels are over for $\mathrm{BC}$ women $(\mathrm{P}=0.02224)$ than normal. Mean glucose levels are inversely linked with insulin $(\mathrm{P}<0.00001)$, interaction effects of leptin and adiponectin (leptin*adiponectin) $(\mathrm{P}=0.08834)$, homeostasis model assessment score insulin resistance (HOMA-IR) and leptin (HOMA-IR*leptin) ( $<<0.00001)$, while they are directly linked with HOMA-IR $(\mathrm{P}<0.00001)$ and leptin $(\mathrm{P}<0.00001)$. The variance of glucose levels is inversely linked with leptin $(\mathrm{P}=0.00022)$, insulin $(\mathrm{P}=0.01365)$, monocyte chemoattractant protein-1 (MCP-1) $(\mathrm{P}=0.01153)$, insulin*HOMA-IR $(\mathrm{P}=0.00022)$, age*resistin $(\mathrm{P}=0.03974)$, age*HOMA-IR( $\mathrm{P}=0.03102)$, while it is directly linked with HOMA-IR $(\mathrm{P}<0.00001)$, resistin $(\mathrm{P}=0.02182)$ and age $(\mathrm{P}=0.00133)$. Glucose levels are higher for $\mathrm{BC}$ women, and they increase along with the increased levels of HOMA-IR, leptin, and the decreased levels of insulin, HOMA-IR*leptin, leptin*adiponectin.
\end{abstract}

Keywords: adiponectin; breast cancer markers; glucose; leptin; MCP-1; resistin; non-constant variance

\section{Introduction}

Worldwide, diabetes mellitus (DM) and cancer are normal diseases with an outrageous impression on human health. Epidemiologic studies present that DM individuals are always at higher risk of cancer [1-4]. Several research reports have illustrated that there is a direct link between obesity and cruelty of $\mathrm{BC}$ [5-7]. A current report has established the linking between $\mathrm{BC}$ markers and body mass index (BMI) [7]. Linking between the $\mathrm{BC}$ prognosis and metabolic syndrome has been recorded in [8-11]. It is noted that metabolic syndrome is acquainted as a summation of at least three of the following metabolic risks such as obesity, high serum triglycerides, elevated serum glucose, higher blood pressure, lower highdensity lipoprotein cholesterol $[8,10]$. The principal mechanisms of the linking between obesity, BC progression and metabolic syndrome have yet to be completely demonstrated, and the previous epidemiological studies remain contradicting [12-16]. A mechanism proposes that it is for higher oestrogen levels that are adjusted from cholesterol [17]. Glucose metabolisms, insulin, resistin, lipid and leptin are postulated as probable intermediate mechanisms that are responsible for developing links between BC markers and obesity [9, 11, 13, 18-20]. A positive linking between triglycerides and $\mathrm{BC}$ markers has been noted in [16]. Also, high insulin and leptin levels are linked with reduced levels of high-density lipoprotein that is supposed to increase risk of BC $[19,20]$.

Cancer research articles are mainly based on preliminary statistics such as usual multiple regression $[8,11,16]$, Cox model analysis [4], logistic regression, Kaplan Meier analysis [20], which are inappropriate for physiological heteroscedastic data analysis. Very few earlier research articles have focused the interaction effects as the component of the linking between glucose levels and BC markers. Recently, some BC marker probabilistic models for resistin, MCP-1, leptin and adiponectin have shown many complicated linkages with glucose levels [21-24]. The current report derives all the findings herein based on the probabilistic models of glucose levels with the BC markers along with the rest factors. The report is arranged as follows. The next section presents materials \& methods, and the subsequent sections present results $\&$ discussions, and conclusions.

\section{Materials and Methods}

\section{Materials}

Study units \& design: 154 Portuguese females currently diagnosed with BC were approved from the University Hospital Centre of Coimbra (CHUC), Gynaecology Department, between 2009 and 2013. The subjects were grouped into four classes depending on their BMI and the BC status. The 4 classes are: (1) BC normal \& BMI levels $<25 \mathrm{~kg} / \mathrm{m}^{2}, \mathrm{n}$ $=29$; (2) BC normal \& BMI levels $>25 \mathrm{~kg} / \mathrm{m}^{2}, \mathrm{n}=48$; (3) BC present with BMI levels $<25 \mathrm{~kg} / \mathrm{m}^{2}, \mathrm{n}=30$; and (4) BC present with BMI levels $>25$ $\mathrm{kg} / \mathrm{m}^{2}, \mathrm{n}=47$. The BC normal, BMI control and obesity study women were taken from the Medicine Department of the same hospital. These 
study subjects were not identified with BC family history and malignant disease.

The selected BC women with normal BMI, or obesity had been treated in the Gynaecology Department of the same hospital. These study subjects were detected $\mathrm{BC}$ for the first time adopting positive mammography and histologically confirmed tests. Moreover, these BC patients were free from any infection, or acute disease during the entry study time. The same doctor researcher extracted all the interested clinical information along with anthropometric data from each selected unit during the first counseling. Finally, only 116 (64 BC and 52 normal women) were taken in the study, and the rest 38 study units were excluded due to obesity with BMI $>40 \mathrm{~kg} / \mathrm{m}^{2}$.

The above illustrated data set can be obtained from UCI Machine Learning Repository, and the detailed description of the data set is presented in $[25,26]$. For necessary application of the factors / variables, they are described as study unit type (SUT) $(1=$ normal; $2=\mathrm{BC}$ patients), age, BMI $\left(\mathrm{kg} / \mathrm{m}^{2}\right)$, Insulin ( $\left.\mu \mathrm{U} / \mathrm{mL}\right)$ (INS), HOMA-IR, Glucose $(\mathrm{mg} / \mathrm{dL})$ (GLU), Resistin (ng/mL) (RES), MCP-1, Adiponectin ( $\mu \mathrm{g} / \mathrm{mL})$ (ADP), Leptin(ng/mL) (LEP).

\section{Statistical methods}

The report aims to derive the probabilistic model of glucose levels based on the remaining as the explanatory variables. It is identified that the response glucose level is heteroscedastic, which should be modeled applying joint generalized linear models (JGLMs) that is clearly presented in [27-29]. It is found that the joint gamma model fit gives better results so it is presented very shortly herein.

\section{Joint Gamma Models:}

Here glucose level $=y_{i}$ say, is the interested study continuous random response variable along with its unequal variance $\left(\sigma_{i}^{2}\right)$, and average $\mu_{\mathrm{i}}$
$=\mathrm{E}\left(\mathrm{y}_{\mathrm{i}}\right)$, obeying $\operatorname{Var}\left(\mathrm{y}_{\mathrm{i}}\right)=\sigma_{i}{ }^{2} \mu_{\mathrm{i}}{ }^{2}=\sigma_{i}{ }^{2} V\left(\mu_{i}\right)$ say, where $V($.$) is$ termed as variance function. Note that the variance has two parts namely, $\sigma_{i}^{2}$ and $V\left(\mu_{i}\right)$, while $V(*)$ characterizes the GLM family distribution. For illustration, if $V(\mu)=\mu^{2}$, it is gamma, and it is Poisson, or Normal obeying as $V(\mu)=\mu$, or $V(\mu)=1$.

Joint mean and dispersion of glucose level $=y_{i}$ models following gamma distribution are presented by

$\eta_{i}=g\left(\mu_{i}\right)=x_{i}^{t} \beta$ and $\varepsilon_{i}=h\left(\sigma_{i}^{2}\right)=w_{i}^{t} \gamma$,

where $g(\cdot) \& h(\cdot)$ are the GLM links attached with the mean \& dispersion predictors respectively, and $x_{i}{ }^{t}, w_{i}{ }^{t}$ are respectively the explanatory variables vectors attached with the mean and dispersion parameters. Maximum likelihood (ML) method is applied to estimate mean parameters, while the restricted ML (REML) method is adopted to estimate dispersion parameters [27].

\section{Statistical and graphical analysis}

The random response glucose level is treated as the dependent variable and the rest others are treated as the explanatory variables. Note that the response glucose level is heterogeneous, which is modeled by JGLMs under gamma distribution. The best model is selected based on the lowest Akaike information criterion (AIC) value that reduces both the squared error loss and predicted additive errors [30, p. 203-204]. A few insignificant effects are added in both the models due to marginality rule introduced by Nelder [31]. JGLMs for glucose level gamma fit outcomes are summarized in Table 1.

\begin{tabular}{|c|c|c|c|c|c|}
\hline \multirow{2}{*}{ Model } & \multirow{2}{*}{ Variables } & \multirow{2}{*}{ Estimate } & \multirow[b]{2}{*}{ s.e. } & \multirow[b]{2}{*}{ t-value } & \multirow[b]{2}{*}{ p-value } \\
\hline & & & & & \\
\hline \multirow{8}{*}{ Mean } & Constant & 4.39334 & 0.02022 & 218.0203 & $<0.00001$ \\
\hline & Insulin (INS) & -0.12821 & 0.00571 & -22.5805 & $<0.00001$ \\
\hline & HOMA-IR & 0.62482 & 0.02843 & 22.0306 & $<0.00001$ \\
\hline & Study unit type (SUT) & 0.02163 & 0.00933 & 2.3203 & 0.02224 \\
\hline & Leptin (LEP) & 0.00351 & 0.00062 & 6.2108 & $<0.00001$ \\
\hline & HOMA-IR *LEP & -0.00174 & 0.00023 & -7.1209 & $<0.00001$ \\
\hline & Adiponectin (ADI) & 0.00092 & 0.00122 & 0.7807 & 0.43713 \\
\hline & LEP*ADI & -0.00013 & 0.00012 & -1.7208 & 0.08834 \\
\hline \multirow{10}{*}{$\begin{array}{l}\text { Disper- } \\
\text { sion }\end{array}$} & Constant & -8.84402 & 1.01915 & -8.6786 & $<0.00001$ \\
\hline & Age & 0.05312 & 0.01607 & 3.3138 & 0.00133 \\
\hline & HOMA-IR & 2.00523 & 0.39254 & 5.1085 & $<0.00001$ \\
\hline & Age* HOMA-IR & -0.00704 & 0.00346 & -2.1865 & 0.03102 \\
\hline & Insulin (INS) & -0.16214 & 0.06472 & -2.5104 & 0.01365 \\
\hline & Leptin (LEP) & -0.03942 & 0.00925 & -4.2906 & 0.00022 \\
\hline & Resistin (RES) & 0.12931 & 0.05533 & 2.3273 & 0.02182 \\
\hline & Age $^{*}$ RES & -0.00212 & 0.00091 & -2.0826 & 0.03974 \\
\hline & INS* HOMA-IR & -0.01132 & 0.00281 & -3.7934 & 0.00022 \\
\hline & MCP-1 & -0.00143 & 0.00052 & -2.5717 & 0.01153 \\
\hline \multicolumn{2}{|l|}{ AIC } & \multicolumn{4}{|l|}{771.8731} \\
\hline
\end{tabular}


The derived glucose level JGLM gamma fit (Table 1) is a data obtained model, so it should be accepted through graphical diagnostic checking, which is revealed in Figure 1. Figure 1(a) presents the glucose level gamma fitted absolute residuals against the glucose level fitted values, which is a flat straight line except right tail. This reveals that variance is equal with the running means. In addition, absolute residuals are

x3

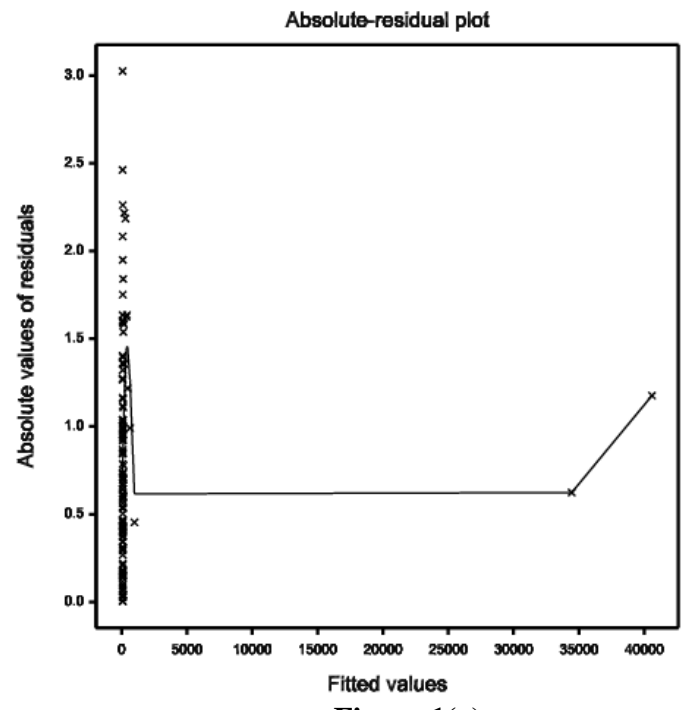

Figure 1(a) randomly dispersed at a point of the fitted value, except only two absolute residuals. Figure 1(b) represents the glucose level gamma fitted mean normal probability plot (Table 1) that does not identify any lack of fit. Figures 1(a) and 1(b) confirm that gamma fitted glucose level JGLMs are close to the true model (Table 1).

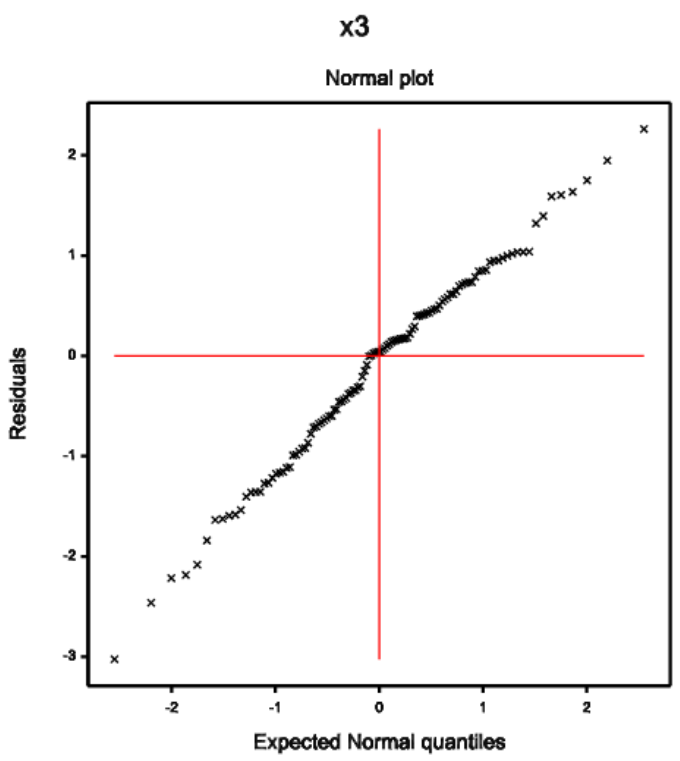

Figure 1(b)

Figure 1: For the glucose level JGLMs gamma fitted (Table 1), the (a) absolute residuals plot against the fitted values, and (b) the normal probability glucose level mean fitted model plot.

\section{Results \& Discussion}

Table 1 reveals the glucose level JGLMs fitted outcomes. It is derived herein that mean glucose levels are over for $\mathrm{BC}$ women $(\mathrm{P}=0.02224)$ than normal. Mean glucose levels are inversely linked with insulin $(\mathrm{P}<0.00001)$, interaction effects of leptin*adiponectin $(\mathrm{P}=0.08834)$, HOMA-IR*leptin $(\mathrm{P}<0.00001)$, while they are directly linked with HOMA-IR $(\mathrm{P}<0.00001)$ and leptin $(\mathrm{P}<0.00001)$. The variance of glucose levels is inversely linked with leptin $(\mathrm{P}=0.00022)$, insulin $(\mathrm{P}=0.01365)$, MCP-1 ( $\mathrm{P}=0.01153)$, insulin*HOMA-IR $(\mathrm{P}=0.00022)$, age*resistin $(\mathrm{P}=0.03974)$, age*HOMA-IR $(\mathrm{P}=0.03102)$, while it is directly linked with HOMA-IR $(\mathrm{P}<0.00001)$, resistin $(\mathrm{P}=0.02182)$ and age $(\mathrm{P}=0.00133)$.

Gamma fitted glucose level mean $(\hat{\mu})$ model (from Table 1 ) is

$\hat{\mu}=\exp (4.3934+0.62842$ HOMA-IR $-0.12821 \mathrm{INS}+0.02163 \mathrm{SUT}+$ 0.00351 LEP

\section{IR*LEP),}

$$
+0.00092 \text { ADI }-0.00013 \text { LEP*ADI }-0.00174 \text { HOMA- }
$$

and the gamma fitted glucose level variance $\left(\hat{\sigma}^{2}\right)$ model (from Table 1) is

$\hat{\sigma}^{2}=\exp (-8.84402+2.00523$ HOMA-IR +0.053 Age -0.00704 Age*HOMA-IR - 0.03942 LEP - 0.16214 INS + 0.12931 RES - 0.01132 INS*HOMA-IR - 0.00212 Age*RES - 0.00143 MCP-1).

From Table 1, it is found that mean glucose levels are directly linked with SUT (1=normal; $2=\mathrm{BC}$ patients) $(\mathrm{P}=0.02224)$, interpreting that glucose levels are over for $\mathrm{BC}$ women than normal. This is frequently noticed in reality, which implies that DM study units have a higher BC risk. Mean glucose levels are inversely linked with insulin $(\mathrm{P}<0.00001)$, concluding that glucose levels decrease as the insulin levels increase. This is the mechanism of balancing glucose levels of human beings. Mean glucose levels are directly linked with HOMA-IR $(\mathrm{P}<0.00001)$, or leptin $(\mathrm{P}<0.00001)$, it concludes that glucose levels increase if HOMA-IR, or leptin levels rise. Even though the HOMA-IR and leptin levels are directly linked with mean glucose levels, their joint interaction effect HOMAIR*leptin $(\mathrm{P}<0.00001)$ is inversely linked with it. In addition, adiponectin $(\mathrm{P}=0.43713)$ is insignificantly linked with mean glucose levels, but the interaction effect leptin*adiponectin $(\mathrm{P}=0.08834)$ is inversely partially linked with mean glucose levels.

The variance of glucose levels is directly linked with age $(\mathrm{P}=0.00133)$ and HOMA-IR $(\mathrm{P}<0.00001)$, while their joint interaction effect age*HOMA-IR $(\mathrm{P}=0.03102)$ is inversely linked with it. These indicate that glucose levels are highly scattered in older women, and also for the subjects with higher HOMA-IR scores. But due to their joint interaction effect, variance of glucose levels is not so highly scattered. In addition, similarly as age, resistin $(\mathrm{P}=0.02182)$ is also directly related with the variance of glucose levels, while their joint interaction effect age*resistin $(\mathrm{P}=0.03974)$ is inversely linked with it. The interpretations are similar to age \& HOMA-IR. Again, HOMA-IR is directly related, and insulin $(\mathrm{P}=0.01365)$ is inversely related with the variance of glucose levels, while their joint interaction effect insulin*HOMA-IR $(\mathrm{P}=0.00022)$ is inversely associated with it. On the other hand, leptin $(\mathrm{P}=0.00022)$ and $\mathrm{MCP}-1$ $(\mathrm{P}=0.01153)$ are inversely linked with the variance of glucose levels, concluding that scatteredness of the glucose levels is higher if the leptin levels, or MCP-1 levels decrease.

From the above it is observed that both for mean and dispersion of glucose levels have very complicated functional linkages with the BC markers such as MCP-1, resistin, HOMA-IR, adiponectin, leptin along with BMI, age and insulin. Note that functional linkages of variance of glucose levels are very little focused in the previous reports. The present findings are 
fully new in the diabetes and BC literature, therefore these outcomes are little reported in the similar earlier published reports.

\section{Conclusions}

The linkages of glucose levels with some BC markers along with BMI, age and insulin are focused in the report based on JGLMs. Note that the accepted gamma fitted model satisfies lowest AIC value, graphical testing, and smaller standard error of the estimates. The current results of glucose levels are very little focused in the earlier reports. It is expected that these linkages of glucose level will be held for any similar data set, which is not verified herein as we have not any similar data in hand. Glucose levels are higher for $\mathrm{BC}$ women, and they increase along with the increased levels of HOMA-IR, leptin, and the decreased levels of insulin, HOMA-IR*leptin, leptin*adiponectin. Medical experts can interpret the role of glucose levels for BC women from this report. Moreover, it may provide very clear activities of glucose levels for the $\mathrm{BC}$ women. DM women should be more careful on BC risk.

Conflict of interest: The authors confirm that this article content has no conflict of interest.

\section{References}

1. Giovannucci E, Harlan DM, Archer MC et al. (2010) Diabetes and cancer. Diabetes Care; 33(7):1674-1685.

2. World Cancer Report. (2008) Boyle P, Bernard L, Eds. Cedex, France, World Health Organization, International Agency for Research on Cancer.

3. Shao S, Gill AA, Zahm SH, Jatoi I, Shriver CD et al. (2018) Diabetes and Overall Survival among Breast Cancer Patients in the U.S. Military Health System. Cancer Epidemiol Biomarkers Prev; 27(1): 50-57.

4. Lopez AD, Mathers CD, Ezzati M, Jamison DT, Murray CJ. (2006) Global and regional burden of disease and risk factors, 2001: systematic analysis of population health data. Lancet; 367:1747-1757.

5. Kulie T, Slattengren A, Redmer J, Counts H, Eglash A, Schrager S. (2011) Obesity and women's health: an evidencebased review. J Am Board Fam Med; 24:75-85.

6. Azvolinsky A. (2014) Cancer prognosis: role of BMI and fat tissue. J Natl Cancer Inst; 106:dju177.

7. Das RN, Lee Y, Mukherjee S, Oh S. (2019) Relationship of body mass index with diabetes \& breast cancer biomarkers. J Diabetes and Management;9(1):163-168

8. Berrino F, Villarini A, Traina A, Bonanni B, Panico S, et al. (2014) Metabolic syndrome and breast cancer prognosis. Breast Cancer Res Treat; 147:159-165.

9. Alegre MM, Knowles MH, Robison RA, O'neill KL. (2013) Mechanics behind breast cancer prevention - focus on obesity, exercise and dietary fat. Asian Pac J Cancer Pre; 14:2207-2212.

10. Antalis CJ, Arnold T, Rasool T, Lee B, Buhman KK, Siddiqui RA. (2010) High ACAT1 expression in estrogen receptor negative basal-like breast cancer cells is associated with LDLinduced proliferation. Breast Cancer Res Treat; 122:661-670.

11. Escrich E, Solanas M, Moral R, Escrich R. (2011) Modulatory effects and molecular mechanisms of olive oil and other dietary lipids in breast cancer. Curr Pharm Des; 17:813-830.

12. Hilvo M, Denkert C, Lehtinen L, Muller B, Brockmoller S, et al. (2011) Novel theranostic opportunities offered by characterization of altered membrane lipid metabolism in breast cancer progression. Cancer Res; 71:3236-3245.

13. His M, Zelek L, Deschasaux M, Pouchieu C, Kesse-Guyot E, et al. (2014) Prospective associations between serum biomarkers of lipid metabolism and overall, breast and prostate cancer risk. Eur J Epidemiol.; 29:119-132.

14. Laisupasin P, Thompat W, Sukarayodhin S, Sornprom A, Sudjaroen Y. (2013) Comparison of serum lipid profiles between normal controls and breast cancer patients. J Lab Physicians; 5:38-41.

15. Melvin JC, Holmberg L, Rohrmann S, Loda M, Van Hemelrijck M. (2013) Serum lipid profiles and cancer risk in the context of obesity: four meta-analyses. J Cancer Epidemiol; 823-849.

16. Rodrigues Dos Santos C, Fonseca I, Dias S, Mendes De Almeida JC. (2014) Plasma level of ldl-cholesterol at diagnosis is a predictor factor of breast tumor progression. BMC Cancer; 14:132.

17. Dumitrescu RG, Cotarla I. (2005) Understanding breast cancer risk - where do we stand in 2005? J Cell Mol Med; 9:208-221.

18. Lambe M, Wigertz A, Garmo H, Walldius G, Jungner I, Hammar N. (2011) Impaired glucose metabolism and diabetes and the risk of breast, endometrial, and ovarian cancer. Cancer Causes Control; 22:1163-1171.

19. Furberg AS, Jasienska G, Bjurstam N, Torjesen PA, Emaus A, Lipson SF, Ellison PT, Thune I. (2005) Metabolic and hormonal profiles: Hdl cholesterol as a plausible biomarker of breast cancer risk. The Norwegian Ebba study. Cancer EpidemiolBiomark Prev; 14:33-40.

20. Melvin JC, Garmo H, Holmberg L, Hammar N, Walldius G, et al. (2017) Glucose and lipoprotein biomarkers and breast cancer severity using data from the Swedish AMORIS cohort. BMC Cancer: 17:246.

21. Kim J, Das RN, Lee Y, Sahoo RK. (2019) Association of monocyte chemoattractant protein-1 with age, glucose, BMI, insulin and other breast cancer biomarkers. Oncology and Radiotherapy; 1 (46):005-009.

22. Das RN, Lee Y. (2019) Association of resistin with BMI, age diabetes and breastcancer biomarkers. Journal of Oncology Research and Treatment; 4(1):135.

23. Das RN, Lee Y. (2018) Association of Serum Adiponectin with Age, BMI and Other Breast Cancer Biomarkers. J Blood Lymph; 8(4):233.

24. Das RN, Lee Y. (2019) Relationship of leptin with glucose, BMI, age, insulin and breast cancer biomarkers. Arch Gen Intern Med; 3(1):01-03.

25. Crisóstomo J, Matafome P, Santos-Silva D, Gomes AL, Gomes M, et al. (2016) Hyperresistinemia and metabolic dysregulation: a risky crosstalk in obese breast cancer. Endocrine; 53(2):433-442.

26. Patrício M, Pereira J, Crisóstomo J, Matafome P, Gomes M, Seiça R, Caramelo F. (2018) Using Resistin, glucose, age and BMI to predict the presence of breast cancer. BMC Cancer; 18(1):18-29.

27. Lee Y, Nelder JA, Pawitan Y. (2017) Generalized Linear Models with Random Effects (Unified Analysis via Hlikelihood) (2nd Ed). London: Chapman \& Hall.

28. Das RN, Lee Y. (2009) Log-normal versus gamma models for analyzing data from quality-improvement experiments. Quality Engineering; 21(1):79-87.

29. Qu Y, Tan M, Rybicki L. (2000) A unified approach to estimating association measures via a joint generalized linear model for paired binary data. Communications in Statistics: Theory and Methods; 29:143-156.

30. Hastie T, Tibshirani R, Friedman J. (2009) the Elements of Statistical Learning, Springer-Verlag, Second Edition.

31. Nelder JA. (1994) The statistics of linear models: back to basics. Statistics and Computing; 4:221-234. 